\title{
Ocho años de ayudas de urgencia ¿para qué?
}

\author{
Eigth years of emergency aid-what for? \\ Agustín Quílez ClaVERo \\ Universidad de Zaragoza (España) \\ 151384@unizar.es
}

Recibido: 17/05/2015

Revisado: $25 / 06 / 2015$

Aceptado: 08/10/2015

Disponible on line: $25 / 11 / 2015$

\begin{abstract}
Resumen
Las ayudas de urgencia son un instrumento del que se vale el Trabajo Social para atender a las personas con recursos económicos insuficientes y, dada la creciente cifra de pobreza, es frecuente tener que utilizarlas. Sin embargo, lejos de considerarlas como un resquicio de una concepción asistencialista, se valora la necesidad de este tipo de ayudas. Por otra parte se piensa que hay que dotarlas de sentido para incorporarlas con normalidad a los acompañamientos en los itinerarios de inserción, puesto que se está viviendo una etapa de cronicidad de problemáticas y de dificultad en el logro de la autonomía personal. Pese a que se trata de una intervención paliativa, no diseñada para el empoderamiento de las personas, el Trabajo Social debe saber interpretar la difícil coyuntura por la que se atraviesa para encontrarle ese sentido.

Palabras clave: pobreza, desempleo, ayuda de urgencia, Trabajo Social, crisis.
\end{abstract}

\begin{abstract}
Emergency-aid programmes are a tool used in social work to attend to people of insufficient financial means. As a result of increasing rates of poverty, they are frequently called upon. However, rather than such programmes being considered a remnant of the welfare-based approach, the need for this type of aid is appreciated. Moreover, it is thought that they must be given meaning in order for them to be incorporated in a regular way into accompaniments for integration programmes as a result of the current period of chronic problems and difficulties in achieving personal autonomy. In spite of the fact that emergency-aid programmes are a form of palliative care and are not designed to empower people, the social work community should be able to chart a path through these difficult circumstances in order to find this meaning.
\end{abstract}

Keywords: poverty, unemployment, social emergency support, social work, crisis.

Referencia normalizada: Quílez Clavero, A. (2015): «Ocho años de ayudas de urgencia ¿para qué?». Cuadernos de Trabajo Social, 28(2): 259-268.

Sumario: Introducción. 1. Metodología. 2. Resultados. 3. Conclusiones. 4. Referencias bibliográficas.

\section{Introducción}

En abril de 2015, en el contexto de un intercambio profesional de trabajadores sociales, el director de la Facultad de Trabajo Social de Viena, Mr. Wilfing, comentaba con resignación que, para encontrar referencias a ayudas que implican alimentación no era preciso consultar un libro de historia de Trabajo Social, ya que se trata de una prestación empleada en nuestros días. En el contexto austriaco, un país con una tasa de paro del 5,8 por ciento, según la Confederación Regional de Empresarios de Aragón (2015), hablar de ayudas de este tipo puede considerarse como algo excepcional o poco frecuente. Pero sí lo es en el caso español, en el cual es habitual el empleo de este tipo de ayudas por los trabajadores sociales que abordan el tema de la pobreza. Se hace por las Organizaciones No Gubernamentales y por las Administraciones Públicas. De hecho, en el momento de redactar estas líneas, el Consejo General de Trabajo Social estaba tratando de dialogar con el Ministerio de Sanidad, Servicios Sociales e Igualdad para que la ayuda de alimentos del Fondo Europeo de Ayuda para los Más Necesitados (FEAD) no se destine de modo indiscriminado a cualquier tipo de asociación. La postura del Consejo de Trabajo Social (2015) consiste en defender que se distribuyera el mencionado Fondo, (que implica ayudas de alimentación) con criterios objetivos. Para garantizar la 
adopción de esos criterios, las trabajadoras sociales ${ }^{1}$ deberían jugar un papel clave, por su rol profesional de evaluadoras y evaluadores de las necesidades sociales individuales. La reunión que se preveía tener, debería tratar de cómo racionalizar la distribución de esos fondos.

Puesto que es un tema muy común en el Trabajo Social, merece la pena estudiar el sentido de este tipo de ayudas. Además de constatar la obviedad del aumento de la demanda de servicios y prestaciones en los Servicios Sociales comunitarios durante los años de la crisis económica, este artículo va a tratar de desvelar qué características sociodemográficas presentan los usuarios que acceden a este tipo de servicios. Por otra parte, a través de la presentación de un caso individual se plantean cuestiones relativas al método del Trabajo Social.

Es necesario, inicialmente, describir brevemente en qué consiste este programa. Aunque puede haber similitudes, a lo largo de la geografía española, es evidente que España es un Estado con diferentes autonomías, en que cada territorio ha adoptado un tipo de servicios independientemente de cómo los organizan otras. Se podría caracterizar esta prestación económica de urgencia, poniendo el ejemplo de una comarca de la provincia de Teruel. En este caso se puede hablar de un programa que atiende a individuos y familias que, de modo puntual, sufren una necesidad. Esta necesidad se encuentra sometida a la comprobación de un/a trabajador/a social y se debe atender de manera inminente. Concretamente se está hablando de ayudas en especie como pueden ser: pagos de recibos de alquiler, de alimentación, compra de electrodomésticos básicos o pagos de recibos de electricidad o agua para evitar cortes de suministro. En el Reglamento de ayudas de urgencia de una comarca del Norte de Teruel se definen este tipo de ayudas como:

Prestaciones económicas de pago único y carácter extraordinario destinadas a resolver situaciones de emergencia que afecten a personas o familias a las que sobrevengan situaciones de necesidad en las que se vean privadas de los medios de vida primarios e imprescindibles, siendo su finalidad el dispensarles una atención básica y urgente en el momento en que aquellas se produzcan (BOPT, 2004).
En España, las comunidades autónomas ofrecen en sus respectivos territorios este tipo de ayudas que el Ministerio de Sanidad, Servicios Sociales e Igualdad (2014) definió como:

Prestaciones de pago único dirigidas a apoyar económicamente a aquellas personas y unidades de convivencia cuyos recursos económicos resulten insuficientes para afrontar situaciones «no previsibles» de necesidad, para hacer frente a gastos específicos de carácter ordinario o extraordinario que cubran necesidades sociales básicas y atiendan situaciones de urgencia o exclusión social.

Siendo este programa eminentemente asistencial, que se focaliza en atender las necesidades primarias, se erige como figura importante en la gestión de esta ayuda el trabajador social. Sin esta figura profesional, este tipo de ayudas podría quedarse en una intervención sin profundidad y sin perspectiva. Se cree preciso dar una oportunidad a las familias de acceder a un trabajador social. Con profesionalidad, este tipo de prestaciones, pueden servir para una intervención más prolongada en el tiempo, con el fin de lograr establecer mayores vínculos entre las personas solicitantes y su entramado social.

\section{Metodología}

La metodología que se ha seguido para elaborar este artículo se fundamenta en dos tipos de técnicas de investigación. En primer lugar, se presenta el análisis de datos que se exponen mediante diferentes gráficos en los que se muestran apuntes sociodemográficos de las personas y las familias que han percibido la ayuda de urgencia en una comarca del Norte de Teruel. Como nota metodológica hay que apuntar que solo se han tenido en cuenta los expedientes con resolución de concesión de la ayuda. Nada se dice de aquellos expedientes archivados o denegados, por una cuestión de unidad muestral. A partir del análisis descriptivo estadístico se muestra la evolución del Programa a lo largo de ocho años. También se apuntan algunas características de las poblaciones que solicitan este tipo de ayudas dado que, por su tamaño manejable, no es necesario trabajar con muestras.

La pretensión de este análisis estadístico no es extrapolar datos ni generalizarlos a partir de

${ }^{1}$ El autor es consciente del androcentrismo del lenguaje y de la alta feminización de esta profesión, pero por mayor claridad expositiva se empleará el masculino genérico. 
una muestra escogida al azar. No se trabaja con muestras ni se pretende dar el salto de la muestra a la población. Se ha tenido cuidado de trabajar con todo el universo, que queda definido por aquellos expedientes administrativos que, en los años 2006, 2009, 2012 y 2014, han sido resueltos con la concesión de una ayuda de urgencia en la citada comarca. La unidad de análisis son expedientes administrativos que corresponden a distintas unidades de convivencia (o si se prefiere hogares). En este análisis estadístico no se habla de personas individuales. Es decir, la unidad de estudio es el expediente administrativo al que corresponden cada año ciertas unidades de convivencia, de las cuales se han estudiado algunas características determinadas. Las conclusiones a las que se llega solo son aplicables a las poblaciones con las que se trabaja cada año. Otra cuestión es que se puedan emplear las conclusiones a las que se llega como hipótesis de partida para estudios posteriores y como ayuda para abrir una vía de investigación, si se aplicara a otras unidades muestrales. Por otra parte, es necesario reconocer que no se han podido establecer comparaciones con estudios similares. Por este motivo se han analizado los datos obtenidos mediante comparación con discusiones teóricas derivadas de la literatura especializada.

Los años escogidos fueron cuatro. El año 2006 se eligió por ser el año previo al estallido de la crisis. El 2009 representa el año al principio de la crisis y el 2012 el año donde la crisis había dejado sus efectos a lo largo del tiempo. El 2014 se escogió por ser el último año con datos disponibles.

Además de la aportación de los datos estadísticos, también se pone el ejemplo de la intervención social realizada y aplicada a un caso concreto. Con la explicación del caso se ilustra la práctica del Trabajo Social con una familia que ha percibido diferentes ayudas de urgencia en el mismo periodo estudiado. No se presenta el caso por su tipicidad, en términos sociológicos, ni tampoco por ser un ejemplo clásico de buenas prácticas, en términos de Trabajo Social, ni se da una especial conexión entre usuario y profesional. Se eligió este caso porque se cree que puede suscitar debate y mostrar las dificultades del profesional en un contexto de tensiones. Así mismo se escogió este caso, y no otro, para poder discutir sobre el difícil equilibrio entre Trabajo Social y servicio social (Pelegrí, 2014). En ocasiones prevalece el servicio sobre el Trabajo Social, lo que no significa necesariamente que la actitud del trabajador social haya sido más proclive a la burocracia que a centrarse en la relación con el otro (Fombuena, 2011). La posibilidad de acceder a la historia social de un caso individual acerca a los lectores a la práctica profesional de un Centro de servicios social de base, servicio de la Administración local enmarcado por el derecho administrativo y muy relacionado con el acceso a las prestaciones.

Con la presentación del caso, se combina el análisis estadístico con otro tipo de datos empíricos, basados en una dimensión cualitativa. Ello fue posible gracias al análisis documental de un expediente administrativo. Se ha escogido un caso en el que se dieron unas condiciones que hic ieron precisa la labor conjunta de la Administración local y las organizaciones no gubernamentales del territorio. Este aspecto se comentará en la discusión de resultados.

\section{Resultados}

\subsection{Análisis descriptivo de datos estadísticos}

Si bien el Trabajo Social de casos se fundamenta en un plan individual para cada uno de ellos, su cuantificación a lo largo del tiempo puede ayudar a ver en perspectiva el valor del volumen del trabajo realizado y su sentido, de los que se trata de reflejar alguna clave en el apartado de conclusiones. Con la aportación de cifras y gráficos se pretende transmitir el valor cuantitativo del trabajo realizado. El principal logro del Trabajo Social se basa en la relación de ayuda, pero también es importante resaltar que esa labor se desempeña con eficacia con un gran número de personas y se constata que la distribución con criterios profesionales de este tipo de ayudas es signo de eficacia y racionalidad.

Como se puede apreciar en el Gráfico 1, el número de expedientes que recoge la concesión de ayuda de urgencia ha aumentado de modo considerable a lo largo de los cuatro ejercicios reflejados, se han quintuplicado, al pasar de 22 a 103 expedientes. Corresponden a núcleos familiares cuyo nivel de renta está por debajo del umbral de la pobreza, ya que para ser elegible por este programa (sin perjuicio de la valoración de la necesidad por parte del trabajador social) las rentas anuales de la unidad familiar no pueden 


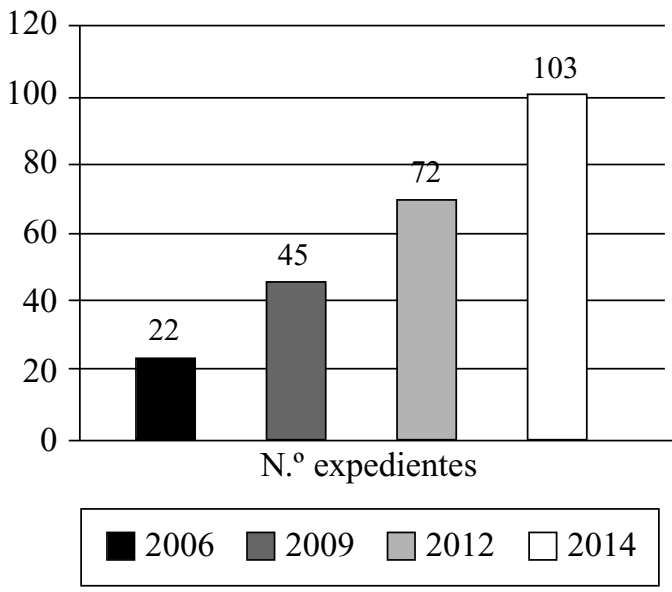

Gráfico 1. N. ${ }^{\circ}$ de expedientes de cuatro ejercicios. Fuente: Elaboración propia.

superar el IPREM, con correcciones al alza del 20 por ciento, según el número de miembros de la unidad familiar.
Por lo que respecta a la nacionalidad de los expedientes de los beneficiarios (teniendo en cuenta que todos los beneficiarios de un mismo expediente tienen la misma nacionalidad), se aprecia una evolución ascendente en ambas categorías. Si bien en 2006 y 2012 eran más numerosos los expedientes de hogares españoles, en 2009 y 2014 son más numerosos los extranjeros. El salto más llamativo es el que se produce entre los años 2009 y 2012, ya que la cifra se duplica en los casos de los extranjeros. También se produce un gran avance en los expedientes nacionales. Las interpretaciones son varias y se tratarán de analizar en la discusión de resultados.

El tipo de convivencia predominante es el de la familia nuclear. Porcentualmente es la forma de convivencia que más aumento ha experimentado. Se ha multiplicado por cinco en un periodo de 8 años. Otras formas de convivencia han experimentado unos incrementos inferiores. Destaca también la cifra de personas solas. El aumento

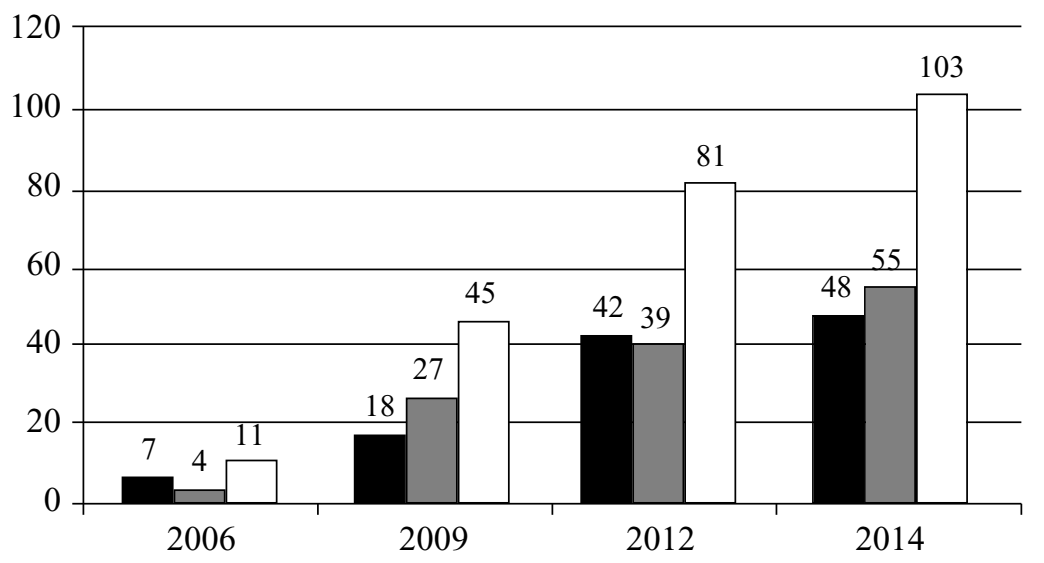

Españoles $\square$ Extranjeros $\square$ Total muestras

Gráfico 2. Nacionalidad de los beneficiarios por expediente.

Fuente: Elaboración propia.

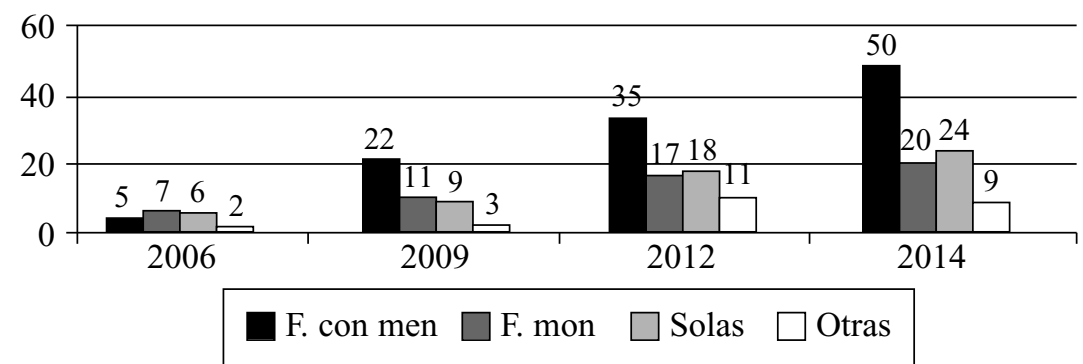

Gráfico 3. Tipo de convivencia.

Fuente: Elaboración propia. 


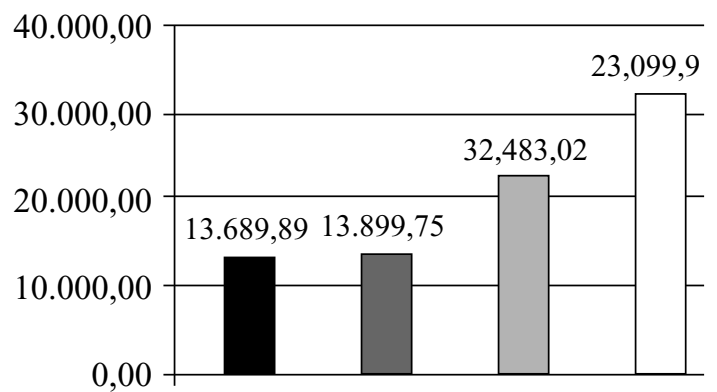

$2006 \square 2009 \quad \square 2012 \quad \square 2014$

Gráfico 4. Evolución del gasto ejecutado.

Fuente: Elaboración propia.

experimentado por ese colectivo se cuadruplica, pasa de 6 a 24. En el apartado de discusión de resultados se buscarán los motivos de este aumento entre las personas solas y también la mayor variación de las familias nucleares.

La evolución del gasto ejecutado también ha sido progresiva. El mayor salto se verifica entre el año 2009 al 2012. Pero también hay otro salto muy notable entre el 2012 y el 2014, que quizá sea más significativo, porque solo han pasado dos años. En comparación con el aumento del número de expedientes en los que se solicitan ayudas, el presupuesto no se ha incrementado en la misma proporción.

\subsection{Descripción de un caso}

El caso que se presenta se refiere a una familia cuya casa sufrió un incendio. Su historia social se abrió en 1989, por cuestiones relativas a la falta de vivienda y las ayudas económicas relacionadas con la carencia de recursos económicos. Se trata de una familia monomarental, tras el divorcio y un episodio de violencia de género, compuesta por la madre de 42 años y tres hijos de 24, 23 y 20 años respectivamente, todos ellos sin formación y sin empleo. Es necesario señalar que, previo a este incidente, también se había registrado en la historia social un episodio de violencia intrafamiliar en la que el hijo de 24 años era el perpetrador y la madre la víctima. En el momento de producirse el incendio (de madrugada), la familia estaba durmiendo en su domicilio y se encontró súbitamente en medio de las llamas. Debido a la rapidez con la que se propagó el fuego, los tres miembros que se encontraban en ese momento en el piso, tuvieron que saltar por el balcón, sufrieron traumatismos y quemaduras, pero su vida no corrió peligro en ningún momento, si bien precisaron hospitalización. El domicilio quedó completamente calcinado lo que les hizo que perdieran sus pertenencias. Si bien el piso era de alquiler (y el más perjudicado económicamente fue el casero), se evidenció una grave situación de necesidad preexistente al incendio, puesto que todos los miembros de la unidad familiar estaban en situación de desempleo.

Una vez fuera del hospital, la familia se dirigió tanto a los Servicios Sociales comunitarios como a Cáritas y a Cruz Roja. Aunque no existe un órgano de coordinación en el territorio que haga converger a estos agentes, para abordar este caso concreto estos tres servicios creyeron conveniente actuar de modo conjunto, para lo cual se mantuvieron reuniones, entre trabajadores sociales y voluntarios de las distintas entidades, y compartieron por ello cierta información. Para posibilitar este tipo de intervención se le preguntó a la familia por la posibilidad de compartir datos y documentación entre las tres entidades indicadas, y la familia accedió. Este acuerdo verbal se cristalizó en la firma de las autorizaciones para la cesión de datos entre las tres entidades, con lo que se evitaba que la familia presentara la respectiva documentación en cada una de ellas.

Desde que salieron del hospital hasta que encontraron un piso de alquiler, los distintos miembros de la unidad familiar se alojaron en domicilios de su familia extensa. El Ayuntamiento de la localidad no tenía ningún programa de alquiler social ni viviendas disponibles en el momento en que ocurrió el incendio como para facilitárselas a la familia. Por otra parte la familia extensa planteó a los Servicios Sociales comunitarios la necesidad de una solución puesto que ellos no podían hacerse cargo del alojamiento de cuatro personas ni a medio ni a largo plazo. La búsqueda de un domicilio (piso de alquiler en el mercado libre) fue muy difícil porque esta familia sufría ciertos estigmas. De hecho, quien escribe este artículo se encontró con la negativa de algún propietario, que acostumbraba a alquilar viviendas, a alquilársela porque adivinaba que podría tratarse de la «familia del incendio». Por el pueblo se había corrido la voz de que no habían pagado nunca un recibo de alquiler del piso que ocupaban (el calcinado). Además habían sufrido un corte de agua por la falta continuada de pago. 
Si bien esta falta de pagos se puede interpretar como una de las consecuencias del desempleo prolongado, algunos signos externos, como la propiedad de vehículos, podían inducir al vecindario a pensar que cierta capacidad económica sí acreditaban.

Según los documentos a disposición de los Servicios Sociales de base, la capacidad económica del grupo familiar presentaba altibajos, pues se alternaban periodos de empleo con periodos de desempleo. Al encontrarnos en un entorno rural, la noticia se difundió entre el vecindario y no fue fácil encontrar un piso en alquiler. Tras varios intentos fallidos, se logró encontrarlo y, a través de las ayudas de urgencia, tanto de la Administración local (tema del artículo) como de Cáritas y Cruz Roja se costearon los primeros gastos para que esta familia pudiera tener un domicilio; concretamente se hicieron tres pagos de la misma cantidad para que dispusiera de un piso de alquiler y de ayuda para alimentación. Los gastos que se asumieron cubrían la fianza y dos meses más, en previsión de que no lograran de modo inmediato un empleo con el que pagar los primeros meses el alquiler.

Mediante el Programa de ayudas de urgencia se siguió apoyando a esta familia con alguna que otra ayuda, puesto que no encontraron trabajo hasta que llegó la temporada de recogida de fruta. Pasaron unos meses en los que carecían de alimentos y otros productos de primera necesidad, lo cual motivó las posteriores ayudas.

Si bien este texto está pensado para evaluar el Programa de ayudas de urgencia en un caso determinado, se ha querido apuntar otros elementos de la intervención para acentuar la necesidad del acompañamiento social en el caso indicado. Se ha pensado así, porque la familia fue especialmente exigente en sus demandas y no gozaban de buena consideración entre el vecindario. Se puede hablar de un caso incómodo. De hecho uno de los hijos acosó y amenazó a la alcaldesa de la localidad por no facilitarle un alojamiento de modo inmediato. Por otra parte, también los demás trataron de amedrentar a un voluntario de Cáritas que colaboró en el caso.

Un año y medio después del incendio, el Ayuntamiento había adecuado dos viviendas con el fin de entregarlas a familias en régimen de alquiler social y, para no adjudicarlas de modo arbitrario, la alcaldesa pidió asesoramiento a los Servicios Sociales comunitarios de la localidad.

\subsection{Discusión de los resultados}

2.3.1. Aumento de demanda y cronicidad de la misma

Como se indicaba en la introducción, la mera constatación del aumento del número de ayudas, por sí misma no aporta gran novedad. Sin embargo refuerza los resultados aportados por otras fuentes de información que ofrecen datos de desempleo y pobreza. Estos pueden consultarse gracias a la Encuesta de Población Activa, el Informe FOESSA o la Encuesta de Condiciones de Vida, año tras año. Unos datos refuerzan a los otros. Las estadísticas que se han elaborado para este artículo tienen correspondencia con otras cifras, como las del aumento de los hogares que reciben renta mínima de inserción en España (Ministerio de Sanidad, 2014). De 2006 a 2012 (de 2014 no se disponen todavía de datos) se pasó de 102.000 hogares a 217.000 hogares con renta mínima de inserción. Se traza una línea ascendente que concuerda con la del gráfico 1. En cuanto a la evolución del gasto de las ayudas económicas de emergencia social (p.8), el incremento es notorio en el territorio español. Concretamente en el caso de Aragón se pasa de gastar 1.900.000 euros en 2008 a 4.100 .000 en 2012. Este aumento de la pobreza ha llevado a algunos autores a hablar de nueva pobreza (Mendiara, 2014), puesto que consideran que se puede denominar así a aquellos que son pobres como consecuencia directa de la crisis económica.

Otra cuestión que se discute es la cronicidad de los casos analizados, que puede tener mayor relevancia a la hora de proponer un plan de intervención social. De los 103 expedientes de 2014 analizados, se repiten 28 hogares que habían recibido también ayuda en 2012, dos años antes. Un tercio de los hogares que recibieron esta ayuda ya la habían percibido antes, lo que significa que siguen en situación de pobreza dos años después. Es criterio para tratar de la pobreza de un hogar no solo que puntualmente se encuentre en esa situación, sino también su cronicidad y estos datos apuntan a la cronicidad.

La división entre extranjeros y nacionales se considera que hay que relacionarla con las cifras demográficas de la zona. Estas cifras (IAEST, 2015) expresan que la población extranjera representa el 12,86 por ciento de la población del territorio objeto de estudio (calculados sobre el 
Censo de 2011). Por lo tanto, que se haya concedido una cifra similar de ayudas a los dos colectivos no es sólo una cuestión cuantitativa sino también cualitativa, y apunta a la hipótesis del mayor nivel de pobreza entre los extranjeros, así como un menor apoyo por parte de sus familias, ya que en muchos casos son aquéllos los que han venido a España para enviar dinero a sus respectivos países.

Sobre el tipo de convivencia, los incrementos experimentados no son iguales en las diferentes categorías estudiadas. Las familias nucleares, categoría más numerosa en cifras absolutas, son las que tienen incrementos más acentuados, se decuplica la cifra de 2006. Las formas de convivencia menos habituales, como los hogares monoparentales o unipersonales, experimentan incrementos moderados, se triplican o cuadruplican. Se podría barajar la hipótesis de que existe una generalización de la precariedad incluso en familias estructuradas, como se sugiere en el Informe FOESSA (2014): «Los hogares que acumulan mayores desventajas son los monoparentales y las parejas con más hijos, lo que refuerza la conclusión previa de que es urgente la mejora de la protección de estas familias» (p. 91)

Una nota aparte merece la categoría «personas solas», que también arroja cifras más altas que la categoría «otros». La cuestión que se comenta es la menor posibilidad de que sea elegible por el Programa de ayudas de urgencia. Encuentra más dificultades para acceder a esta ayuda aquella unidad familiar en cuyo seno haya algún conviviente con un salario o una pensión. La unidad de convivencia formada por una sola persona es más proclive a tener este tipo de ayudas si no supera un monto económico anual, aunque podría obtener un apoyo informal igual o más importante que el de quienes conviven bajo el mismo techo.

\subsubsection{Análisis de un caso individual}

La coordinación como factor estratégico:

Respecto al caso individual expuesto, se pueden debatir varias cuestiones. En primer lugar se destaca, como buena práctica, el empleo de la coordinación para atender a la familia. La literatura especializada se decanta por un trabajo social coordinado: «Ningún servicio de urgencias sociales podría gestionar directamente recursos suficientes para realizar por sí mismo e íntegramente todas estas funciones. La coordinación con otros agentes es imprescindible» (Duque, 201, p. 77).

En este caso se evitó que la familia tuviera que hacer tres gestiones diferentes y visitar tres agencias distintas para obtener prestaciones. Por otra parte, sirvió para organizar y racionalizar los gastos y para aumentar la magnitud de las prestaciones materiales para la familia. Fueron los Servicios Sociales comunitarios quienes asumieron el rol organizador. Sin embargo el mecanismo de coordinación fue creado ad hoc, al no existir ningún mecanismo reglado de coordinación en el territorio.

\section{¿Nuevos pobres?}

¿Son nuevos pobres o viejos pobres? Según alguna autora (Mendiara, 2014, p. 74) serían nuevos pobres, y cito: «las personas que han presentado una demanda relacionada con la crisis económica». Si bien la relación profesional se desencadenó con el hecho puntual del incendio, la familia presentada estaba muy orientada al mundo laboral. En primavera, los tres hermanos encontraron trabajo (temporal) en la recogida de fruta, lo que sirvió para paliar su problemática económica. Por el contrario, la madre con una ligera discapacidad física, inferior al 33 por ciento, no ha trabajado más de dos meses desde la apertura de la historia social, lo que la encuadraría en la categoría de pobre de larga duración. No es una mera discusión terminológica, probablemente la precariedad les siga persiguiendo, pero en esta familia se observó una actitud muy activa en la búsqueda de empleo. La tendencia a la cronicidad vendría dada más bien por la falta de oportunidades que por la falta de actitud.

En busca de ética, profesionalidad, autenticidad: Otra cuestión que puede suscitar debate es la intervención en casos especialmente incómodos para el profesional, como se cree que fuera el que se expone. Por una parte, se produjo una evidencia en la necesidad, puesto que un incendio es una desgracia manifiesta; pero por otra parte, esta familia, cuyo caso era conocido por los diferentes trabajadores sociales de la localidad - Cáritas, Cruz Roja o Servicios Sociales de base - no despertó mucha solidaridad. Puede ser contradictorio, pero es lo que ocurrió. Evidentemente los sentimientos que suscitan las familias deben ser tamizados por el filtro de la profesionalidad, ya que empatizar con la familia es una 
actitud profesional aconsejada por manuales (Azpeitia, 2003); pero se pone de manifiesto que esos sentimientos se despiertan y hay que gestionarlos. Para ello es especialmente recomendable el trabajo de equipo. Con la intervención de un grupo profesional se pueden gestionar con mayor mesura dos tipos de intervención. Por un lado, se amortigua el exceso de actividad que suscita un caso de gran carga emocional, y por otro lado se puede ayudar a evitar la falta de implicación o apatía. En particular, se ha presentado una familia especialmente exigente en sus demandas, que no facilitó una relación de confianza. Si bien ni la entidad local ni la regional tenían previsto un programa de vivienda para este tipo de emergencias (lo cual pudo provocar una decepción), su familia más próxima tampoco se mostró especialmente activa para solucionar el problema. El historial de conflictividad de este grupo familiar pesó en el apoyo informal recibido y en la intervención social que se le prestó.

El reto pasa por explorar qué pueden hacer tanto los técnicos de atención directa, como los responsables organizativos de los servicios, para prevenir este tipo de situaciones; programando actuaciones de seguimiento que se adelanten en lo posible a las crisis que desencadenan en muchas ocasiones intervenciones urgentes (Santos Santos, Chans Pousada, Cantero Saiz y Hamparzoumian Montilla, 2014, p. 78)

En resumen se proponen dos cuestiones para el debate: por una parte, la falta de previsión de los servicios oficiales de vivienda y, por otra parte, cómo intervenir con un tipo de familia incómoda, que genera unos sentimientos que dificultan la empatía que debe practicar el profesional (Azpeitia, 2003). La situación de crisis o la petición de una ayuda económica es una oportunidad para establecer una relación de ayuda, como diría Santos: «Aprovechar las crisis de las familias para generar una relación de ayuda» (Santos Santos, Chans Pousada, Cantero Saiz y Hamparzoumian Montilla, 2014, p. 78). Pero en ocasiones no se generan los condicionantes para poder hacerlo en toda su profundidad, como sucedió en este caso.

Si se asumen los supuestos de autoras, como Josefina Fombuena-Valero o Alfonsa Rodríguez, se debe procurar una relación auténtica con los usuarios y superar la de la simple aplicación de la fórmula automática necesidad-recurso. Pero en este caso concreto, hay que reconocer abierta- mente que no fue posible iniciar un proceso de acompañamiento social con profundidad. Los motivos por los que no se pudo hacer se han esbozado ya, a lo largo del artículo. En primer lugar habría que hablar de las características de la familia. Previamente esta familia ya trató de obtener la renta mínima de inserción, falseando la situación de convivencia, lo cual puede dar una idea de lo que espera de las instituciones esta familia. Lejos de volver a enjuiciarla o de partir de los prejuicios que se tienen de la misma, el comportamiento de los Servicios Sociales de base se mantuvo dentro de unos parámetros de comprensión ante una desgracia evidente. Por otra parte, la familia no estaba en condiciones de iniciar esa relación por el estrés que produce haber atravesado una situación crítica, como es un incendio, y por tener preocupaciones distintas a la hora de expresar sentimientos e inquietudes ante un trabajador social (a lo que nunca se mostraron proclives). La familia precisa de soluciones inmediatas que, en el caso analizado, en lugar de propiciar una relación profesional de acercamiento, lo que provocó fue el tratar las cuestiones concernientes a las necesidades más básicas como: solucionar el problema de alojamiento y la manutención. El trabajo social quedó en segundo plano y pasó al primer plano el servicio social, la prestación, el derecho administrativo.

Como consideración final queda la respuesta genérica de la Administración local ante un problema de vivienda de cualquier vecino en situación de emergencia. Si bien, en un principio no se contaba con viviendas sociales ni con un programa de alquiler social, el incidente del incendio tuvo una consecuencia positiva: se movilizó la sensibilidad de la Administración local y, en cierto modo empujada por este incidente, fueron acondicionadas dos viviendas de propiedad municipal para apoyar a familias con problemas de alojamiento. El hecho de pedir asesoría a los Servicios Sociales de base para elaborar una convocatoria pública de alquiler social, muestra la confianza de la autoridad local en este servicio: se confió en nuestra labor, más allá de que se tuviera en consideración que faltan asesores en los ayuntamientos pequeños. Se debe valorar este hecho porque finalmente se asumieron las propuestas de los Servicios Sociales de base a la hora de redactar la mencionada convocatoria. Se elaboraron unas bases para que cualquier familia pudiera optar a esas viviendas. Una propuesta de 
convocatoria (se recuerda que en el medio rural se dan mecanismos de coordinación informales con frecuencia) fue redactada por los Servicios Sociales comunitarios. Como criterios de adjudicación tuvieron en cuenta elementos de discriminación positiva para favorecer a familias con ciertas dificultades; es decir, se contemplaron circunstancias como: haber sido víctima de violencia de género, estar en situación de discapacidad, haber sufrido desahucio o catástrofe en la vivienda, ser familia numerosa o estar en paro todos los miembros de la unidad familiar en el momento de presentar la solicitud. La familia cuyo caso se ha presentado formuló su solicitud y logró acceder a un alquiler social. En el momento de redactar estas líneas todavía ocupa la vivienda de alquiler social del Ayuntamiento.

Se considera que esos criterios tienen un sentido claramente social que en cierto modo ayudaron a vincular una actuación puntual de urgencia con una intervención social a más largo plazo $\mathrm{y}$, sobre todo, con una filosofía que entiende la acción social. Por otra parte se vio reconocida la labor del Trabajo Social del territorio.

\section{Conclusiones}

Diferenciar entre asistencialismo y asumir la creciente necesidad de conceder ayudas para gastos básicos es importante para el Trabajo Social. Pese a tener preferencia por concepciones del Trabajo Social preventivas, que traten de evitar las causas que originan la pobreza, no se puede dejar de atender las emergencias, es decir la situación evidente. No se considera que exista contradicción desde el punto de vista de la intervención social individual. En la actualidad la pobreza severa es un tema que se ha de abordar con apoyos materiales, sin desdeñar por ello la importancia de establecer una relación con los usuarios más profunda.

También se debe señalar que la tendencia a la cronicidad de ciertos casos debe mantenernos alerta en dos sentidos: en primer lugar, se debe comprender que no es sencillo que los usuarios logren la autonomía en contextos de alto desempleo y, por lo tanto, no alcanzar esta autonomía, en muchos casos, no es achacable a la falta de actitud por su parte. Con ello se quiere apuntar que los procesos de inserción social se pueden alargar. Por el otro, y pese a lo indicado anteriormente, las relaciones de dependencia entre el usuario y los Servicios Sociales deben evitarse a largo plazo, ya que ciertos usuarios podrían acercarse a situaciones de cronicidad llevados, probablemente, por un cierto desánimo.

En esas aguas pantanosas se mueve el Trabajo Social, aguas pantanosas, a las que alude la profesora Alfonsa Rodríguez (2006) cuando describe su propuesta de método de trabajo, en el que se deben matizar la cientificidad, el protocolo, o la asepsia. La intervención puede ser tan simple como facilitar unos alimentos a una familia o tratar de hacer una intervención más profunda y cualitativa si el usuario es proclive a ello. Cuando se tiene esa oportunidad - no es siempre posiblehay que dar pie al usuario a que exprese sus inquietudes y (se) plantee soluciones a sus propias necesidades. También se precisa en ocasiones dejar al usuario que se exprese, sin tratar de llegar de modo inmediato a la elaboración de un plan de intervención. En ese ambiente propicio que posibilita a la persona expresarse, el trabajador social ha de tomar buena nota de lo acontecido para iniciar un auténtico proceso de acompañamiento. Por el contrario en contextos de alto estrés y burocracia (Ballestero e Idareta, 2013; Miranda y Vilas, 2013) no es fácil para el profesional encontrar esos espacios de encuentro. En esos contextos, el poco tiempo disponible y gran volumen de trabajo no permiten ni propician un prolongado diálogo con los usuarios. Visto de ese modo, el Trabajo Social se ve reducido a una mínima expresión y puede caer engullido por las exigencias de los Servicios Sociales (Pelegrí, 2014).

Finalmente, en cuanto a la actitud de los profesionales ante casos individuales incómodos, se han apuntado dos cuestiones en la discusión de resultados y se quieren resaltar como conclusiones. Para este tipo de casos, es necesario el tamiz de la profesionalidad. Por otra parte, se precisa la aportación y supervisión de un equipo de trabajo que refuerce la labor de la persona que tiene asignado el caso. No se requieren cantidades ingentes de tiempo para esta labor de equipo si está bien organizada. Por lo tanto es necesario que se creen, o se mantengan, estos espacios de reflexión y apoyo mutuo. Pese a padecer un contexto de alto estrés hay que pensar que el equipo supone un antídoto frente a la carga emocional del profesional. Habría que favorecer tiempos y espacios para facilitar reuniones de equipo, más allá de creer que otro tipo de ocupaciones más tangibles, como las de gestión de recursos, son las únicas importantes y las únicas que representan eficacia. 


\section{Referencias bibliográficas}

Azpeitia et al. (2003). Manual para el trabajo social de acompañamiento en los itinerarios de inserción. Madrid: Colegio Oficial de Trabajadores Sociales.

Ballestero, A. e Idareta, F. (2013). Ética, burocracia y paternalismo en Trabajo Social. Portularia 12 (1), 27-35.

Cañedo, M. (2011). ¿Y esto a quién se lo cuento, al ayuntamiento?: Trabajadores sociales entre la vocación y la burocracia. Un análisis antropológico del modelo de intervención de los Servicios Sociales a partir de la etnografía de una red asistencial madrileña. Cuadernos de Trabajo Social, 24, 135-153. http://dx.doi.org/10.5209/rev_CUTS.2011.v24.36864.

Consejo General de Trabajo Social. (2015). Comunicados de prensa. Recuperado de: http://www.cgtrabajosocial.es/noticias/el-consejo-general-y-los-36-colegios-profesionales-de-trabajo-social-piden-una-reunion-al-ministro-de-sanidad-servicios-sociales-e-igualdad-para-trasladar-sus-lineas-rojas-respecto-al-fead/2843/view (Consultado el 1 de octubre de 2015).

CREA. (2015). Tasas de desempleo estimado. Países de la Unión Europea. Disponible en: http://www.crea.es/economia.nsf/dd4f2d53559dbb62c1256aa0005b23f2/8cc5e2bb7576f6d4c125 6b4300629396? OpenDocument (Consultado el 11 de septiembre de 2015).

Díaz-García, O. (2011). El trabajo social ante a la crisis. Portularia, número extraordinario, 239-244.

Duque, T. (2011). Acción social municipal en situaciones de urgencia y emergencia social. Experiencia de las capitales de la CAPV. Zerbitzuan, 49, 71-81.

Fombuena, J. (2011). ¿Quién es el otro del Trabajo Social? Alteridad y Trabajo Social. Portularia, 11(2), 61-68.

FOESSA. (2014). VII Informe sobre exclusión y desarrollo social en España. Madrid: Fundación FOESSA/Cáritas.

IAEST. (2015). Información estadística territorial. Andorra-Sierra de Arcos. Disponible en: http://www.comarcas.es/pub/documentos/documentos_27_ANDORRA_057797c7.pdf (Consultado el 17 de mayo de 2015).

Mendiara, C. (2014). Las redes de apoyo social de la nueva pobreza atendida en el Centro Municipal de Servicios Sociales del Barrio de la Magdalena de Zaragoza. Revista Portularia. 14(1), 73-86.

Ministerio de Sanidad, Servicios Sociales e Igualdad. (2014). Informe de rentas mínimas de inserción 2013. Recuperado de: http:/www.msssi.gob.es/ssi/familiasInfancia/inclusionSocial/serviciosSociales/RentasMinimas/Docs/Informe_2013.pdf. (Consultado el 13 de mayo de 2014).

Miranda, M. y Vilas, L. (2013). Estudio sobre la profesión de Trabajador social en Aragón. Colegio Profesional de Trabajo Social de Aragón. (Inédito).

Pelegrí, X. (2014). Trabajo social y servicios sociales: una complementariedad diferenciada. Notas para el cambio de una época. Acciones e Investigaciones Sociales, 34, 7-24.

Boletín Oficial de la Provincia de Teruel. (2004). Reglamento comarcal de Ayudas de Urgencia de la Comarca de Andorra-Sierra de Arcos. Boletín Oficial de la Provincia de Teruel. 236.

Rodríguez, A. (2006). El proceso de integración en la teoría y la práctica en la docencia de Trabajo Social con familias. Recuperado de: http://dialnet.unirioja.es/servlet/autor?codigo=1230756

Santos Santos, L., Chans Pousada, J.C., Cantero Saiz, A. y Hamparzoumian Montilla, I. (2014). Trabajo Social en un centro de Servicios Sociales: una familia con 70 gatos. Cuadernos de Trabajo Social, 27(1), 71-80. http://dx.doi.org/10.5209/rev_CUTS.2014.v27.n1.43170 